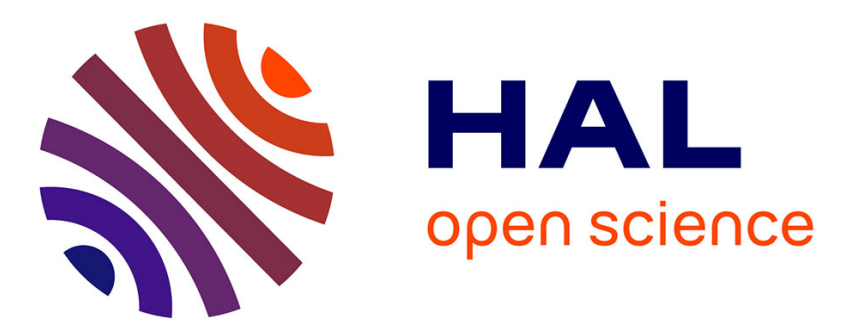

\title{
Characterizing plastics originating from WEEE: A case study in France
}

Elisabeth Maris, Pierre Botane, Philippe Wavrer, Daniel Froelich

\section{To cite this version:}

Elisabeth Maris, Pierre Botane, Philippe Wavrer, Daniel Froelich. Characterizing plastics originating from WEEE: A case study in France. Minerals Engineering, 2015, 76, pp.28-37. 10.1016/j.mineng.2014.12.034 . hal-01206310

\section{HAL Id: hal-01206310 https://hal.science/hal-01206310}

Submitted on 28 Sep 2015

HAL is a multi-disciplinary open access archive for the deposit and dissemination of scientific research documents, whether they are published or not. The documents may come from teaching and research institutions in France or abroad, or from public or private research centers.
L'archive ouverte pluridisciplinaire HAL, est destinée au dépôt et à la diffusion de documents scientifiques de niveau recherche, publiés ou non, émanant des établissements d'enseignement et de recherche français ou étrangers, des laboratoires publics ou privés. 


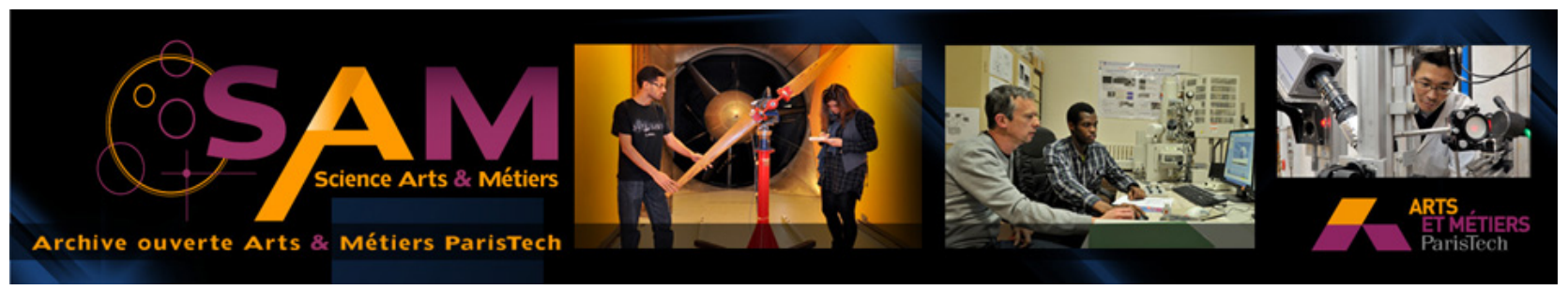

\section{Science Arts \& Métiers (SAM)}

is an open access repository that collects the work of Arts et Métiers ParisTech researchers and makes it freely available over the web where possible.

This is an author-deposited version published in: http://sam.ensam.eu

Handle ID: .http://hdl.handle.net/10985/10203

\section{To cite this version :}

Elisabeth MARIS, Pierre BOTANE, Philippe WAVRER, Daniel FROELICH - Characterizing plastics originating from WEEE: A case study in France - Mineral Engineering - Vol. 2015, $n^{\circ} 76$, p.28-37 - 2015 


\title{
Characterizing plastics originating from WEEE: A case study in France
}

\author{
Elisabeth Maris ${ }^{a, *}$, Pierre Botané ${ }^{b}$, Philippe Wavrer $^{c}$, Daniel Froelich ${ }^{a}$

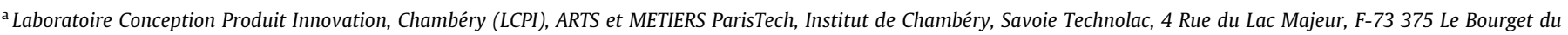 \\ Lac Cedex, France \\ ${ }^{\mathrm{b}}$ CASPEO, 3 avenue Claude Guillemin, BP 36009, 45060 Orleans Cedex 2, France \\ ${ }^{\mathrm{c}}$ BRGM, 3 avenue Claude Guillemin, BP 36009, 45060 Orleans Cedex 2, France
}

Keywords:

Sampling procedure

Characterization procedure

Polymers

Waste

WEEE

\section{A B S T R A C T}

Two studies, TRIPLE (For Analyse des gisements DEEE et optimisation des technologies de TRI des PLastiques DEEE (analysis of WEEE and optimization of sorting technologies for WEEE plastics).) and VALEEE (For VALorisation des composants, matières et substances issus du gisement DEEE (Recovery of components, materials and substances from WEEE).), supported by the French State, the Greater Lyon area (Grand-Lyon), the Rhône-Alpes Region and the French eco-organization "Eco-systèmes", and involving laboratories, recycled material users and recycler partners, were conducted concerning the characterization, sorting and recovery of French WEEE (Waste Electrical and Electronic Equipment).

To determine the heterogeneity of a 10-ton batch, the WEEE was sorted into families before grinding. Specimens were dismantled and plastic particles were analyzed to estimate their composition. The batch was then crushed and the metals extracted.

The residue containing plastics was sampled at the outlet of the plant and analyzed. The detailed characterization of the plastics sample was used to calculate the estimated sampling error and the overall measurement error. The sample size was determined so as to achieve satisfactory accuracy for the most represented polymers likely to be recovered after recycling.

A simple characterization methodology for use by recycling plants was proposed in order to determine the plastic composition of this waste. The procedure was validated on a second 10-ton batch of sWEEE collected from another location and treated by a different recycling facility.

This article presents the sampling protocol design methodology, then the characterization protocol and its usage limitations.

\section{Introduction}

The quantity of plastics in Waste Electrical and Electronic Equipment (WEEE) has increased significantly over the past few years. In order to specifically manage this waste, the European Commission has implemented the Directive 2002/96/EC (Huisman et al., 2008), setting targets for WEEE recovery between $70 \%$ and $80 \%$. This quota range cannot be fulfilled by the state-ofthe-art metal and glass recycling methods. Hence, for environmental and financial reasons it is becoming imperative to recover most of the plastics from WEEE. Apart from the regulatory aspect, recycling plastics from WEEE preserves raw material resources and reduces energy consumption (80-90\% of the energy can be saved by using recycled materials versus raw materials - BIR, 2009).

Although WEEE is collected in five different streams (cooling appliances, TVs or computer monitors, large household electrical equipment and small waste electric and electronic equipment), this

\footnotetext{
* Corresponding author. Tel.: +33 (0) 479253 663; fax: +33 (0) 479253670

E-mail address: elisabeth.maris@ensam.eu (E. Maris).
}

study focuses on the Small Waste Electric and Electronic Equipment (sWEEE) category represented for instance by IT (computers, phones, printers, etc.), culinary equipment, audio/video, vacuum cleaners, hand electric tools, personal care products and toys.

For sWEEE, the performance of the plastics recycling processes highly depends on its composition (Chancerel and Rotter, 2009). Recycling-oriented characterization is therefore a systematic approach to support the design and operation of recycling processes. Plastics must be separated before being remolded due to the immiscibility between certain polymers (Froelich et al., 2007) or due to prohibition of hazardous substances (European Directive RoHS 2002/95/CE).

Two studies, TRIPLE $^{1}$ and VALEEE, ${ }^{2}$ supported by the French State, the Greater Lyon area (Grand-Lyon), the Rhône-Alpes Region

\footnotetext{
${ }^{1}$ For Analyse des gisements DEEE et optimisation des technologies de TRI des PLastiques DEEE (analysis of WEEE and optimization of sorting technologies for WEEE plastics).

${ }^{2}$ For VALorisation des composants, matières et substances issus du gisement DEEE (Recovery of components, materials and substances from WEEE).
} 


\section{Abbreviations}

ABS acrylonitrile-butadiene/styrene

$\mathrm{PC}+\mathrm{ABS}$ polycarbonate $+\mathrm{ABS}$ blend

PET polyethyleneterephthalate

PA polyamide

PP polypropylene copolymer

PP-T20 polypropylene $+20 \%$ talc

LDPE low density polyethylene

RoHS Restriction of Hazardous Substances regulation
WEEE Waste Electrical and Electronic Equipment

PMMA polymethylmethacrylate

PVC polyvinylchloride

POM polyoxymethylene

PS polystyrene

TPU thermoplastic polyurethane

PE polyethylene and the French eco-organization "Eco-systèmes", and involving laboratories, recycled material users and recycler partners, were conducted concerning the characterization, sorting and recovery of French sWEEE. One objective of these two studies was to provide a method of characterization to be used routinely by recycling plants to determine the plastic composition of this waste. Indeed, to our knowledge, there is currently no such a method for a simple and rapid characterization that allows operators to check the composition of the crushed plastics from sWEEE.

In order to determine the heterogeneity of the sWEEE, 10t of it were sorted into families before grinding. Specimens were manually sorted and dismantled and the plastic particles were analyzed to estimate their composition. The batch was then crushed and the metals extracted.

The residue containing plastics was sampled at the plant outlet and analyzed. The detailed characterization of the plastics sample was used to calculate the estimated sampling error and the overall measurement error. The sample size was determined so as to achieve satisfactory accuracy for the most represented polymers likely to be recovered after recycling. The procedure was validated on a second 10-ton batch of sWEEE collected from another location and treated by a different recycling facility.

This article presents the sampling protocol design methodology, then the characterization protocol and its usage limitations.

\section{Sampling methodology}

\subsection{Characterization of the batch}

The current study focuses on the composition of a batch of plastic residue that is a by-product of a metal recycling facility after crushing, grinding and metal recovery (Fig. 1).

Two batches (representing a total of about 10t) of sWEEE were identified before being treated by the metal recycling facility. Their characterization was conducted at the feed and by-product ends of the process, in order to be able to link the characterization of the plastic by-products to a composition in terms of equipment types.

For each sWEEE batch, each piece of equipment was sorted manually by category: IT (computers, phones, printers, etc.), culinary equipment, audio/video, vacuum cleaners, hand electric tools, personal care products and toys. Each category was weighed. From each one, three specimens were selected at random and dismantled; a total of 67 items were dismantled. The polymer parts obtained were weighed and analyzed using Mid InfraRed (MIR) spectroscopy. Table 1 presents the composition of the categories in terms of polymers. This composition is calculated as the average of the three specimens. A rough estimate of the polymer composition of the batch is indicated by the weighted average and presented in Fig. 2.

This characterization method was used in order to have (1) an idea of the types of polymers that could be found, and (2) a rough estimate of their frequency. Due to the wide heterogeneity of the pieces of equipment inside each category, the composition obtained constitutes qualitative information that must compared with the characterization of the plastic residue.

In order to characterize the batch of this SWEEE fraction, a sample of the by-products of the plastic residue was collected, by taking ten increments distributed over the batch treatment period. Taking advantage of the fact that the batch was in movement on a belt conveyor, and to reduce the effect of distribution heterogeneity due to segregation, the ten increments were collected at the discharge of this conveyor, resulting in a $9.3 \mathrm{~kg}$ primary sample.

After shredding, the plastics particles passed $40 \mathrm{~mm}$. Since the objective of the sampling procedure was to quantify the recyclable fraction of plastics, only the $+20 \mathrm{~mm}$ fraction that could be processed by automatic/optical sorting machines had to be characterized. Then, the $9.3 \mathrm{~kg}$ primary sample was screened at $20 \mathrm{~mm}$. The non-polymer particles in the $+20 \mathrm{~mm}$ fraction were manually extracted, identified and weighed. Inside the remaining $5.5 \mathrm{~kg}$, about 1500 polymer particles were counted, weighed and analyzed one by one using MIR spectroscopy for 21 different polymers. Fig. 3 shows a comparison between the plastics composition of two batches.

The results of this detailed characterization were used to set up a heterogeneity model allowing calculation of the sampling error, which is the first step in the sampling procedure design.

\subsection{Sampling procedure design}

It is possible to calculate the fundamental sampling error based on the heterogeneity model, following the Pierre Gy sampling theory (Gy, 1979). The heterogeneity model consists in a description of the material heterogeneity related to the parameter to be measured, i.e. in our case, the polymer composition.

In particulate material sampling, the randomly selected elements are particles, and in this case pieces of plastic. These particles can be classified into homogeneous families of similar particles with respect to their mass and their composition. In our case, the plastic particles are made of one polymer with a $100 \%$ content for that polymer and $0 \%$ for the others. Indeed, the particles made of a composite of several polymers were sufficiently rare to avoid taking them into account here.

As for the sampled particles in the $20-40 \mathrm{~mm}$ size range, their mass is in a sufficiently narrow range to constitute a quasi-homogeneous family. Each polymer is then represented by one family and the polymer content is no more than the family's proportion in the batch. Thus, the relative variance of the fundamental sampling error (FE) for the measurement of the batch's polymer composition is given by the Pierre Gy formula (Eq. (1)):

$\left.\sigma^{2}(F E)=\left(\frac{1}{M_{S}}-\frac{1}{M_{L}}\right) \quad m_{c} \frac{1-2 t_{c}}{t_{c}}+\sum_{i=1}^{N_{F}} m_{i} t_{i}\right)$

where $M_{S}$ is the mass of the sample, $M_{L}$ the mass of the initial batch, $t_{i}$ the fraction of the family, $i$ in the sample, $t_{c}$ the fraction of the family, $c$ which is also the fraction of the considered polymer, $m_{i}$ 


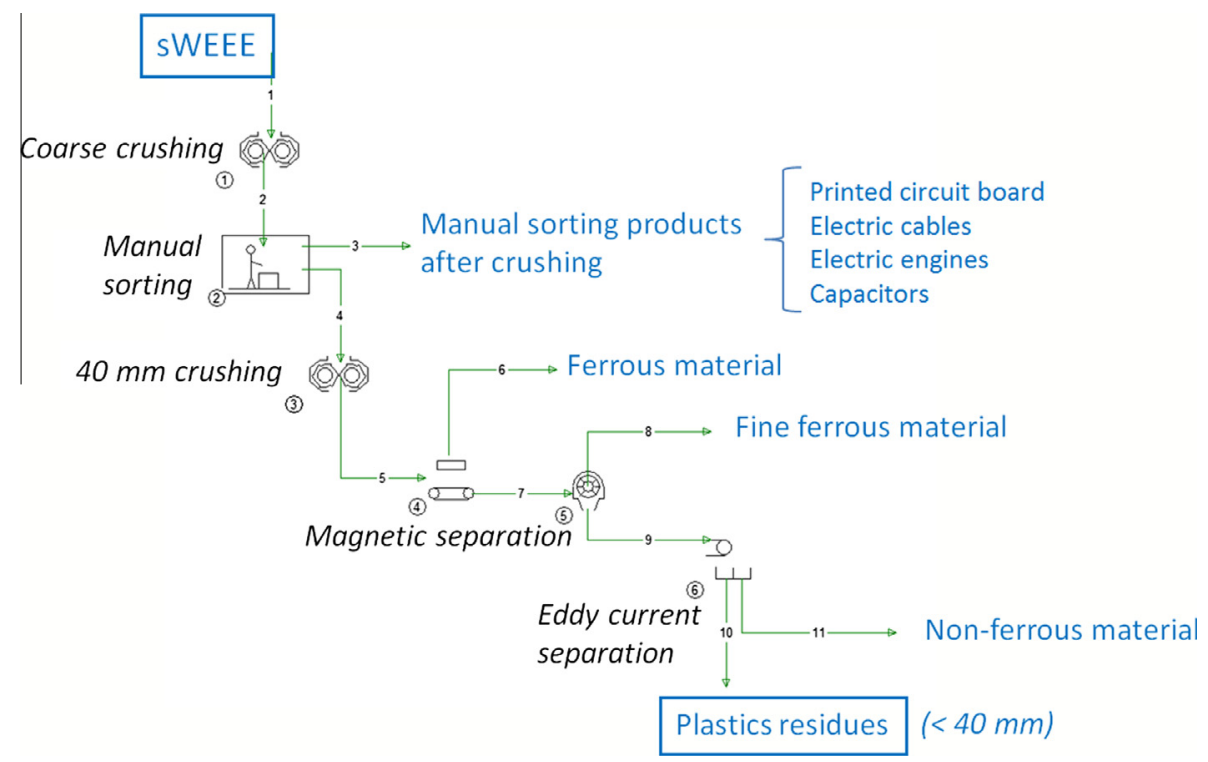

Fig. 1. The sWEEE treatment process.

Table 1

Mean polymer composition of the sWEEE equipment categories (in mass\%).

\begin{tabular}{|c|c|c|c|c|c|c|c|}
\hline Polymers ${ }^{\mathrm{a}}$ & IT & Culinary & Audio/video & Vacuum & Hand tools & Personal care & Toys \\
\hline ABS & 21.6 & 18.9 & 40.7 & 49.4 & 7.0 & 62.0 & 60.3 \\
\hline HIPS & 49.8 & 0.2 & 36.9 & & & & 33.8 \\
\hline PP & & 67.0 & 1.2 & 30.8 & 70.5 & 5.2 & \\
\hline PC-ABS & 11.6 & 0.2 & 0.2 & 6.2 & & 6.0 & \\
\hline PMMA & 5.9 & 2.1 & 1.7 & & & 0.6 & \\
\hline PC & 4.4 & 3.7 & 0.6 & 1.7 & 5.4 & 0.8 & \\
\hline PA & 0.1 & 2.7 & 1.5 & 2.9 & & 5.0 & 1.0 \\
\hline Others & 6.6 & 5.1 & 17.2 & 9.0 & 17.1 & 20.3 & 4.8 \\
\hline
\end{tabular}

a ABS: Acrylonitrile Butadiene Styrene; HIPS: High Impact Polystyrene; PP: Polypropylene; PMMA: Poly(methyl methacrylate); PC: Polycarbonate; PA: Polyamide.

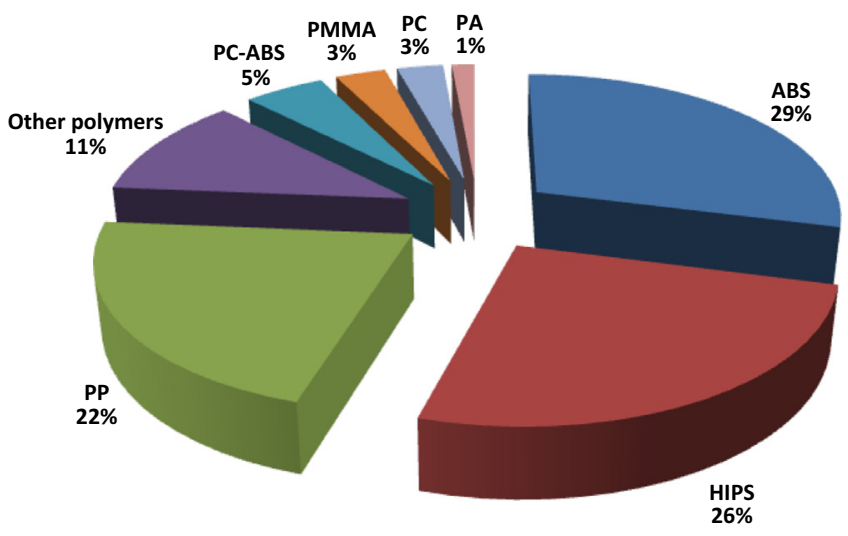

Fig. 2. Estimate of the polymer composition of the $10 \mathrm{t}$ batch of sWEEE.

the mean unit mass which is the mean mass of all the particles in the family $i$.

Since the particles in the $20-40 \mathrm{~mm}$ range were analyzed one by one, they were manually classified into polymer families. The mean unit mass of each family is easily obtained by weighing the entire family and dividing the weight by the number of particles.

Since the sample analysis is only performed on the $+20 \mathrm{~mm}$ fraction, the batch is, in fact, the $+20 \mathrm{~mm}$ fraction of the plastic residue for a given period of production. It can be considered to be the portion of the sWEEE constituted of polymer particles that can be industrially sorted for potential recycling. Despite this restrictive definition of the batch, its mass (several tons) can be considered as very large compared to the sample mass. The variance of the fundamental sampling error is then inversely proportional to the sample mass and is a function of the polymer fraction. Applying Eq. (1) to the $5.5 \mathrm{~kg}$ of collected $+20 \mathrm{~mm}$ particles, the variance of the fundamental sampling error can be calculated for each polymer and is indicated in Fig. 4 through the error bars corresponding to the $95 \%$ confidence interval.

The less represented polymers have a large relative fundamental sampling error and their numbers of particles in the sample are between 1 and 15 . For these polymers, the assumptions made to obtain Eq. (1) are not verified and the 'Poisson effect' occurs (François-Bongarçon, 2009). As these polymers have no recycling advantage, the measurement of their proportion was not considered for the procedure design.

Conversely, the sample quantity to be collected to reach our accuracy objective can be calculated from Eq. (1). Fig. 4 presents the fundamental sampling error associated with the measurement of the polymer composition as a function of the sample mass. The less represented polymers are not displayed, as their error appears above $100 \%$. For the most frequent and valuable polymers - ABS, HIPS, PP, PC-ABS and PC - the fundamental sampling error was considered as acceptable by final users with a $5.5 \mathrm{~kg}$ sample.

\subsection{Sampling procedure}

The first step before applying the sampling procedure is to clearly define the batch. In our case, where sampling took place 


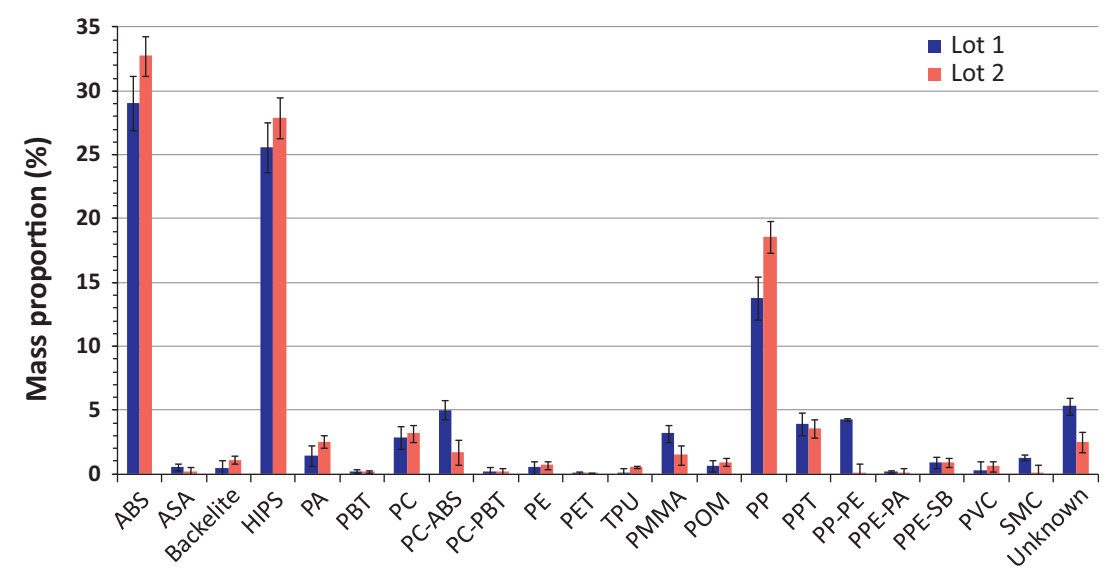

Fig. 3. Plastics composition of two batches of shredded sWEEE and the associated uncertainty.

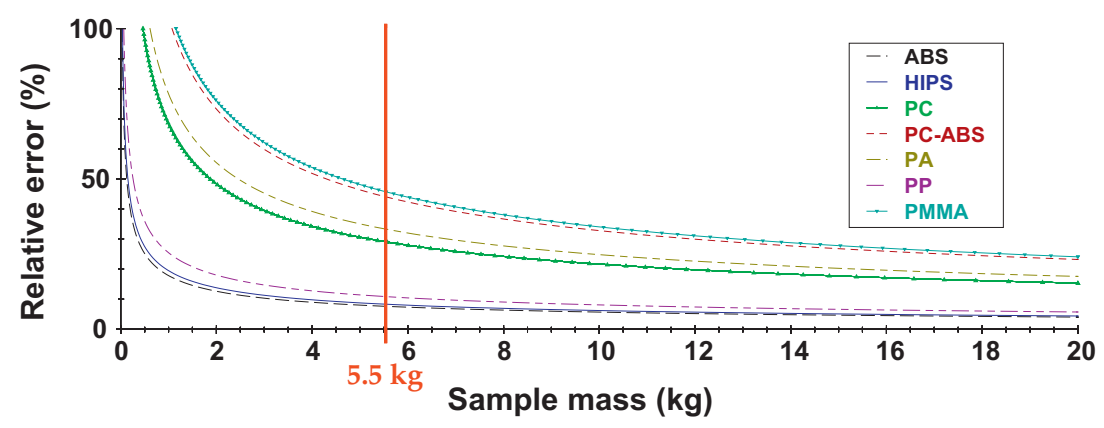

Fig. 4. Fundamental sampling error depending on the size of the sample.

on a stream, the batch was characterized by a treatment period. For example, this period can be a production period such as 1 day or, here, the period to treat a batch of a given shredded sWEEE.

During the considered period, 10 increments were collected in order to minimize the long range quality fluctuation error due to the distribution heterogeneity. The resulting sample is then a composite of increments distributed over the entire period. To avoid contamination by the remaining material in the grinding facility and abnormal treatment conditions, the starting and the ending phases of the operation during the collection period were excluded.

The ten increments were collected at the discharge of a conveyor, which is the best location for continuous sampling operations (Holmes, 2008). Special attention was paid to the fact that segregation occurs during conveying (fines migration and sticking of particles) and that the material is not homogeneously distributed at the discharge. A cross stream cutter was used, taking care to cut the entire stream to respect the equiprobability assumption: each batch particle must have the same probability of being sampled.

Since the increment was performed manually, the following rules were complied with (Fig. 5):

- The sampling container shape was rectangular, with a width of at least three times the coarsest particle size $(>3 \times 40 \mathrm{~mm}=$ $120 \mathrm{~mm}$ ) and a sufficient length $L$ to add $100 \mathrm{~mm}$ on both sides of the falling stream section: $L>l+200 \mathrm{~mm}$. The container capacity was at least twice the expected volume of collected increment, and it had a sufficient depth to avoid particle loss due to rebound.

- The container was moved horizontally and linearly, parallel to the conveyor roller, in a single pass, through the material flow, at a constant speed. The speed was determined to obtain approximately $1 \mathrm{~kg}$ after one pass under the stream.
- The increments were sampled as close as possible to the conveyor end, where the flow is generally less dispersed and the falling speed is low.

- The container was emptied after each increment collection.

All of the increments were mixed to obtain the composite sample. The sample was then screened to remove the fines particles $<20 \mathrm{~mm}$ that were not recoverable. The coarse fraction was then analyzed as described previously.

A compromise between representativeness and analysis cost had to be achieved. A quantity of about $5.5 \mathrm{~kg}$ of coarse fraction was considered acceptable by all of the project partners. This quantity corresponds to a $10 \mathrm{~kg}$ composite sample.

\subsection{Sampling methodology discussion}

The accuracy obtained from the characterization of the $9.3 \mathrm{~kg}$ sample is considered satisfactory by the project partners (then the different users of the plastic particles) for the most represented polymers (ABS, HIPS, PP, PC-ABS and PC), although this accuracy is significantly worse for the less represented polymers. It is important to take into account that the entire final sample must be analyzed in order to guarantee this accuracy. Any reduction in the sample mass results in a dramatic increase of the sampling error, resulting in less representativeness and confidence in the analytical results. Conversely, considering the sampling error, the primary composite sample mass can be increased. It may be the case when the residue flow rate is high and then when much more than $1 \mathrm{~kg}$ is collected for each increment. It may be also the case when the collection period is long and when many more than 10 increments have to be collected, in order to minimize the distribution heterogeneity. In these cases, the primary composite sample has to be perfectly-homogenized and then divided using dedicated devices 


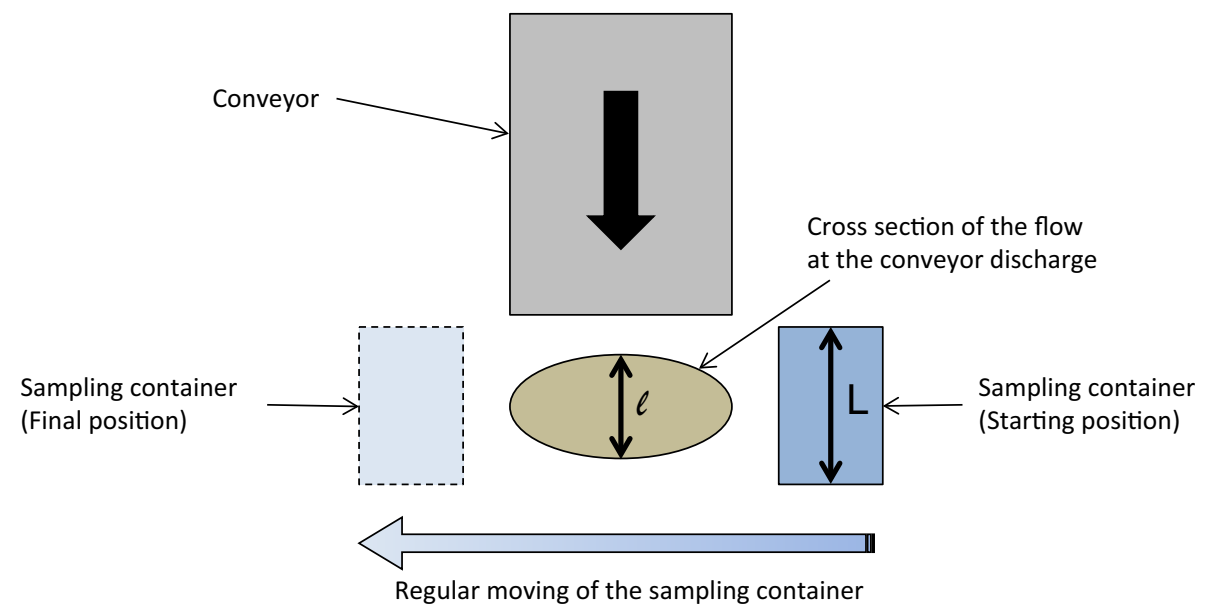

Fig. 5. Sampling procedure used at the discharge of the conveyor.

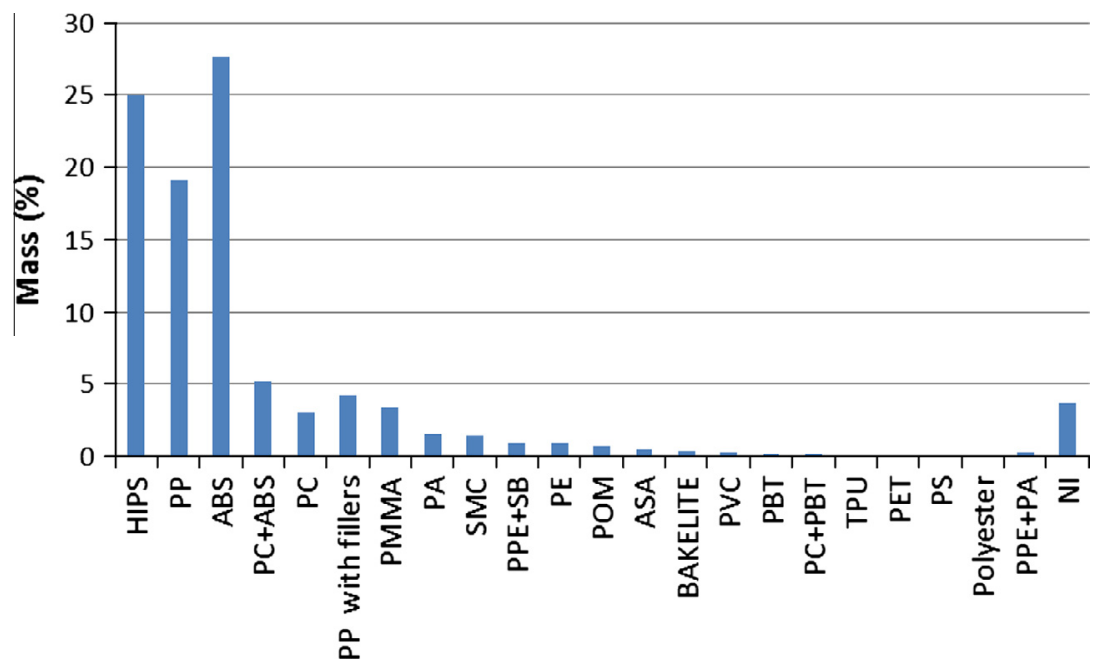

Fig. 6. Composition and mass distribution of polymers from the representative WEEE fraction sample.

to obtain at least a $10 \mathrm{~kg}$ final sample. It is this $10 \mathrm{~kg}$ sample that will be screened at $20 \mathrm{~mm}$.

The major limitation in this procedure is the extrapolation of the composition measured for the $+20 \mathrm{~mm}$ fraction to the $-20 \mathrm{~mm}$ fraction. As it has been observed that the polymers do not have the same size distribution after the grinding stage, this extrapolation cannot be done. Since the polymer particles finer than $10 \mathrm{~mm}$ are difficult to recycle using optical sorting or other processes, restricting measurement to the particles coarser than $20 \mathrm{~mm}$ is economically justified here.

\section{Polymer characterization methodology}

It is essential to learn more about plastic waste composition and contaminants in order to perform a cost-effective recovery of the materials in compliance with the regulations.

The plastics in the reference sample were analyzed according to the following criteria: type of polymer, nature and quantity of flame retardant and fillers and additives (Bromine, Cadmium, Chromium, Lead, Chlorine, Antimony, Silicon, Phosphorus, Aluminum, Titanium, and Magnesium), density, color, grinding and sorting defects. The type of polymer was characterized using Mid-infrared (MIR) spectrometry, whereas the nature and quantity of flame retardants and fillers and additives were characterized using X-ray fluorescence spectrometry.

\subsection{Grinding and sorting defects}

The grinding defects resulted from links still existing between two or more materials after the grinding step. The sorting defects were due to materials other than polymers. Contaminants were extracted through manual sorting. They included paint coating, the remaining links between plastic parts or between plastic parts and a different material, and other types of materials such as glass or electronic cards. The grinding and sorting defects impact the quality of the recycled polymer.

\subsection{Polymer analysis using mid-infrared spectrometry}

All of the plastics were analyzed with a Bruker Mid-infrared spectrometer (used in specular reflection) to identify the polymer families. Unlike the NIR analysis, it is possible to identify blackcolored polymers with a laboratory MIR device. Although no particular preparation of the analyzed samples is necessary, they must be flat and larger than $10 \mathrm{~mm}$. The spectrum of the analyzed samples is compared with that of a library of spectra of roughly 20 families of polymers and their additives. This library is based on the analysis of several standards of polymer families containing different additives and corresponding to various applications. During the analysis, the device identifies the polymer matrix and indicates the spectral distance that represents the degree of 


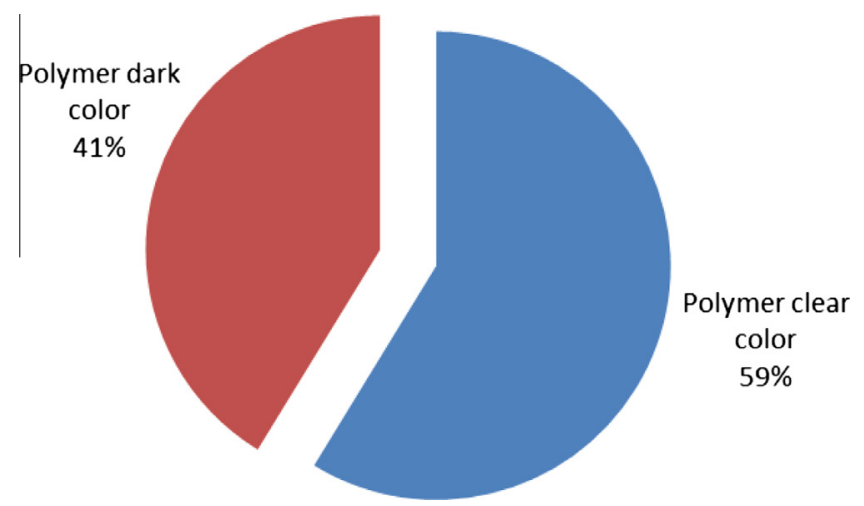

Fig. 7. Percentage of light and dark polymers from WEEE's.

similarity with a spectrum from the library. A spectral distance of 0 indicates that both spectra are identical. This value increases when the difference between spectra increases. Samples are classified as "unidentified" when the spectral distance is superior or equal to 1 . This value is determined as being the threshold below which the identification of the spectrum is correct with a confidence interval of more than $90 \%$.

\subsection{Color}

The color of the plastics is a determining criterion for plastics sorting using an industrial NIR (close infrared) spectrometer. Only light-colored plastics are recognizable with this technology. This technology is suited to plastics that cannot be sorted out by density separation when their densities overlap (Photo 1).

\subsection{Fillers and flame retardants: fluorescence-X detection}

A BRUKER S2 RANGER spectrometer was used for the X-ray fluorescence analyses. This device can detect the elements Sodium to Uranium. Using a specific method, it is also possible to deduct compounds such as mineral fillers (talc, chalk or glass fibers) from the elementary analysis. The results are interpreted using a semiquantitative analysis and a quantitative method depending on the elements. These methods take into account the effects of the various polymers (Compton signal) and the inter-element influence. The results are obtained with a minimum relative error of 5\%. A standard (Standard NF 62321 (01/06/2009) 2009) describes the contaminant analysis protocol required by the RoHS (Restriction of Hazardous Substances Regulation), but no standard

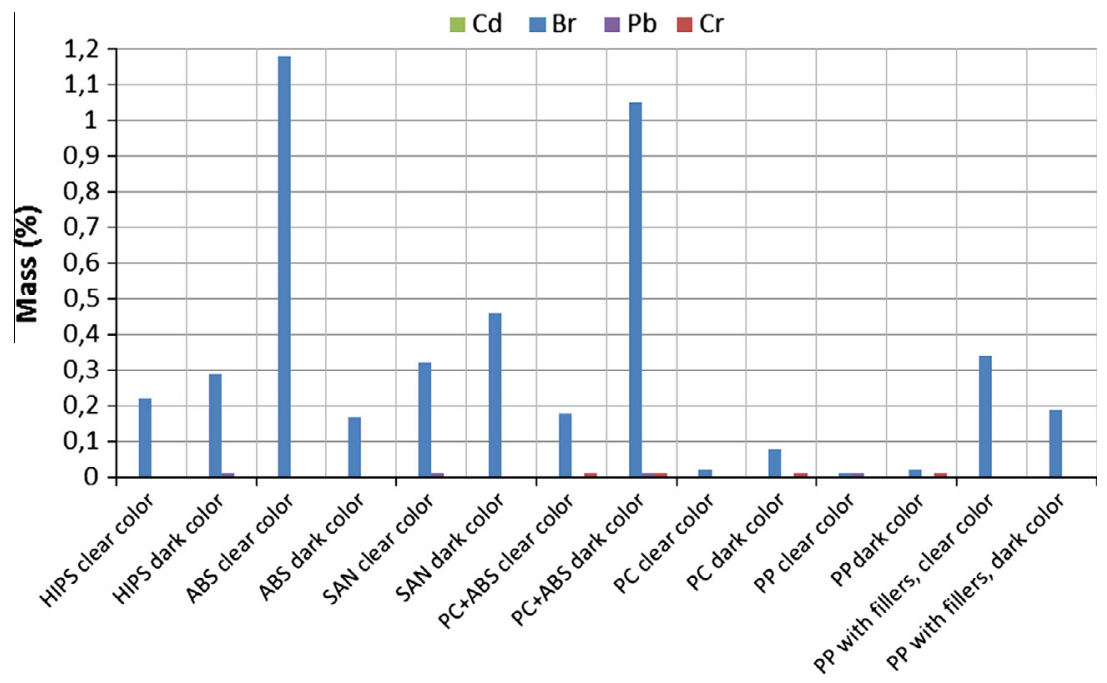

Fig. 8. Concentration of regulated substances.

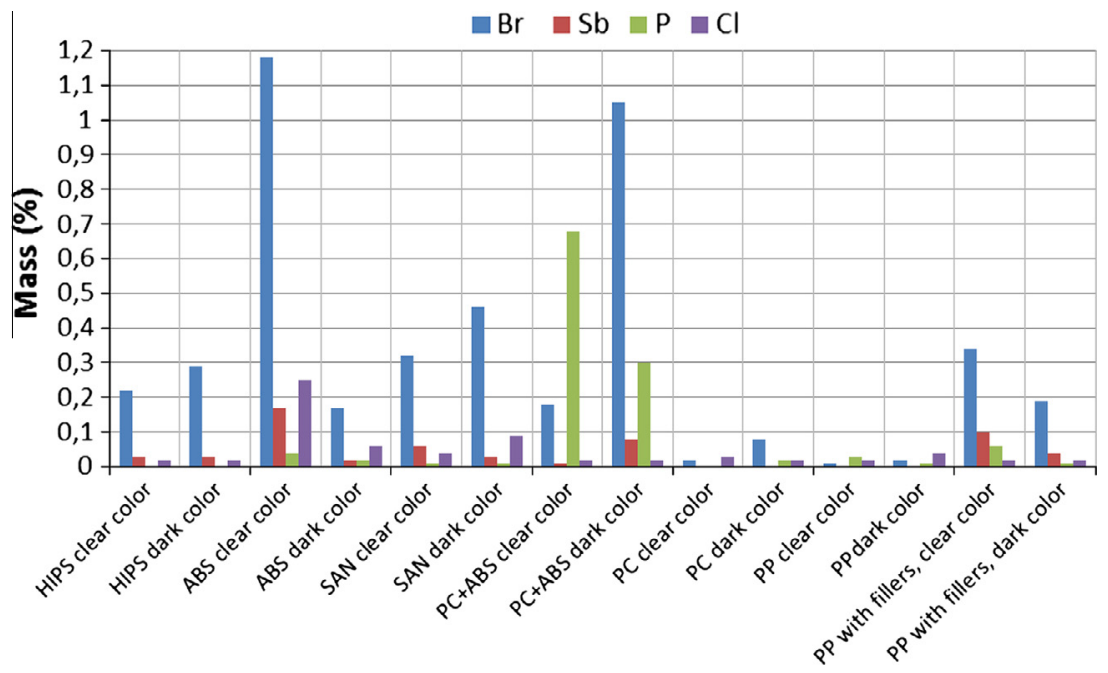

Fig. 9. Tracer concentration of flame retardants. 


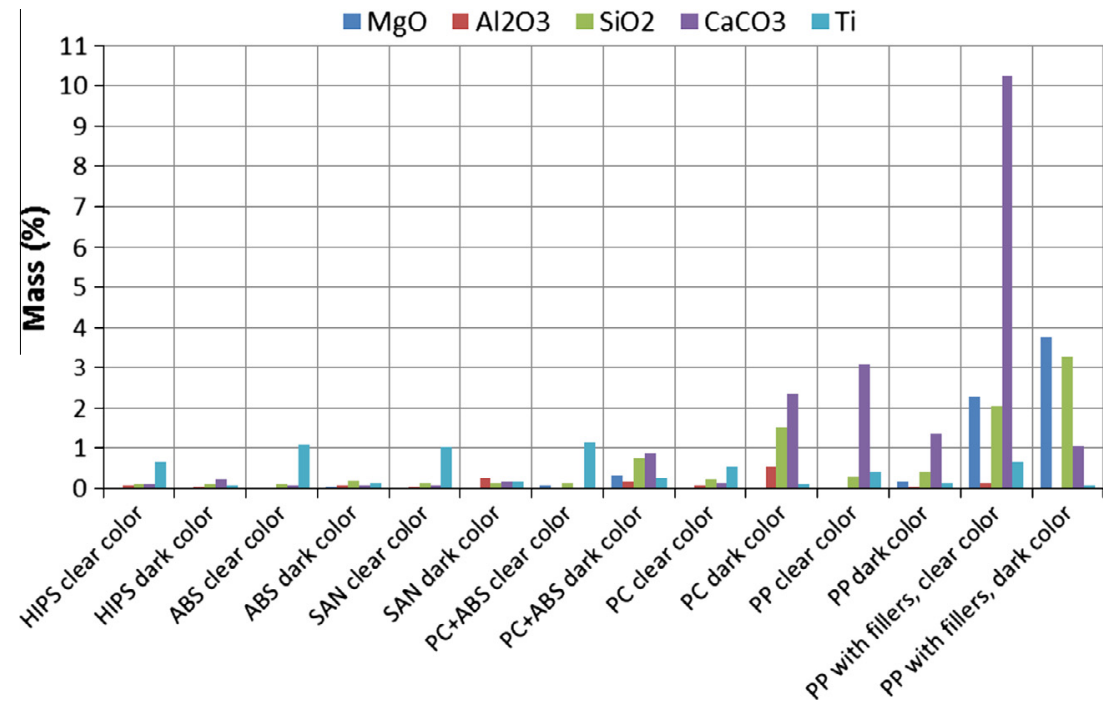

Fig. 10. Concentration of fillers for each fraction of polymers

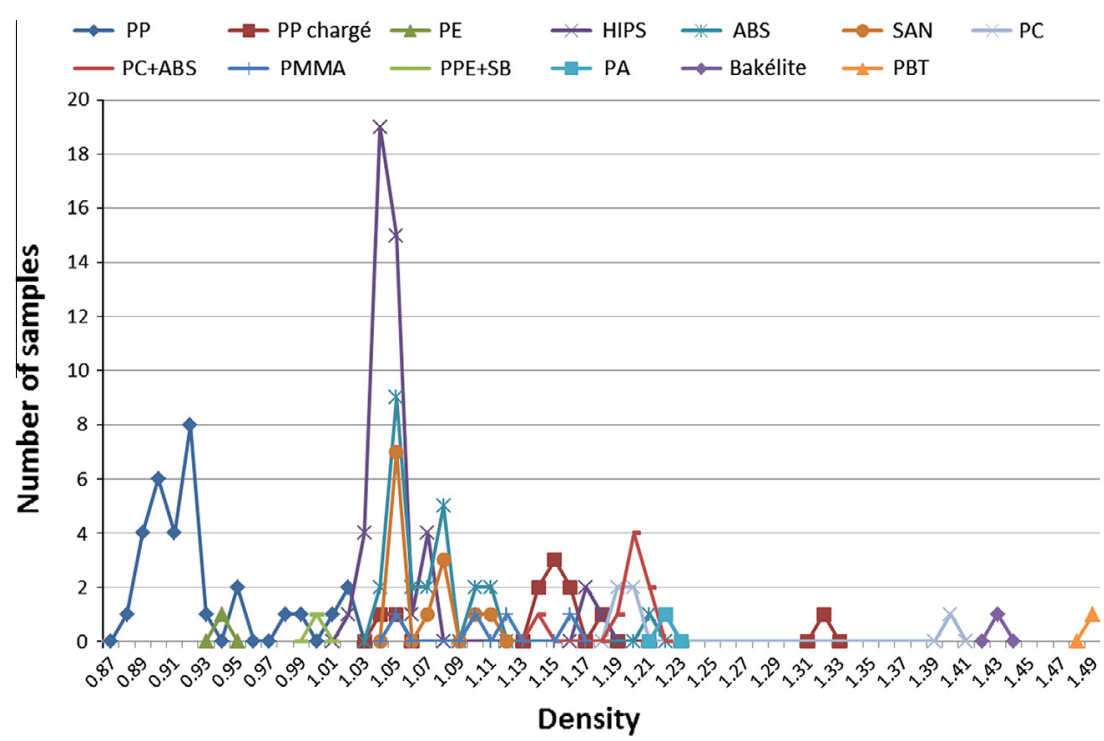

Fig. 11. Density distribution of polymers.

describes a sampling and analysis protocol according to the European WEEE regulations (WEEE Directive, 2003).

A procedure was set up with all of the research program partners, whereby the quantities of substances are measured in the fractions of polymers sorted out by family. Only the main families, represented by more than $4 \%$, were analyzed. To be representative, the fractions of each family were reduced to powder by micro grinding to the average size of $1 \mathrm{~mm}$. After homogenization of the powders, two $10 \mathrm{~g}$ samples were taken. These samples were then analyzed using X-ray fluorescence with a detection threshold set at $100 \mathrm{ppm}$ by the quantitative method.

The elements analyzed were: $\mathrm{Br}, \mathrm{Cl}, \mathrm{P}, \mathrm{Ca}, \mathrm{Sb}, \mathrm{Si}, \mathrm{Al}, \mathrm{Mg}, \mathrm{Cr}, \mathrm{Cd}$, $\mathrm{Pb}$ and $\mathrm{Ti}$. The concentrations in $\mathrm{Br}, \mathrm{Cl}, \mathrm{P}$ and $\mathrm{Ca}$ were determined by means of a calibration established according to ABS standards. The calibration curves were based on 2 or 3 standards with a minimal concentration in $\mathrm{Br}, \mathrm{Cl}$ and $\mathrm{P}$ of $5 \%$ (The standards concentration was controlled by Inductively coupled plasma mass spectrometry). Consequently, the relative error on the concentration values below $5 \%$ is unknown. The semi-quantitative method was used to determine the concentration of all the other elements. The relative precision for the measurements was $5 \%$. The presence of fiberglass is characterized by the elements $\mathrm{SiO}_{2}, \mathrm{Al}_{2} \mathrm{O}_{3}$ and $\mathrm{CaO}$ in certain proportions. Fillers such as talc (magnesium silicate) are characterized by the presence of magnesium and silicon, whereas kaolin (aluminum silicate) is characterized by the presence of aluminum and silicon. Calcium carbonate is characterized by the presence of the calcium element only. Flame retardants are characterized based on their atomic compositions and associated molecules.

The methodology of calculation with Bruker software includes two types of calculation. First the results in the raw data form related to mass percentage of each element are analyzed. The sum of all the elements must be close to $100 \%$. Secondly the software allows recalculating this sum in molecules and oxide form. In this case, on the basis of realistic assumptions, formulas of target molecules are entered. The assumptions of mixed molecules are validated when the calculated sum are close of $100 \%$. For example if the elements $\mathrm{Mg}$ and $\mathrm{Si}$ be not $\mathrm{Al}$, are found but not $\mathrm{Al}$, the 


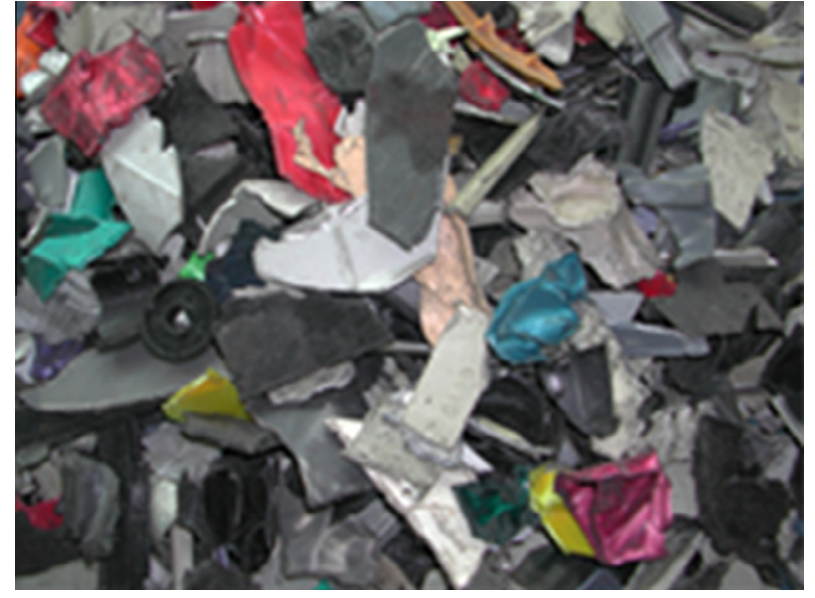

Photo 1. Samples of polymers originating from WEEE's.

formula of magnesium silicate is entered. If the sum of all the molecules is $100 \%$, the result is validated. If $\mathrm{Al}, \mathrm{Si}$, and $\mathrm{Mg}$ are found the two formulas of aluminium silicate and magnesium silicates are tested with different percentage and are validated when the sum is $100 \%$. If not the formula of trihydrate de magnesium is tested. The presence of antimony for example validated the presence of bromine from flame retardants even in small concentration. Clearly MIR, X-rays diffraction analysis can also give information but these methods are not enough efficient for concentration calculation.

Table 2 presents the tracers of substances as well as flame retardants and the mineral fillers associated with these tracers. The substances regulated by the RoHS directive are hexavalent chromium, lead, mercury, cadmium, polybromobiphenyls (PBB) and polybromodiphenylethers (PBDE). The maximal concentration tolerated is $0.01 \%$ for cadmium and $0.1 \%$ for the other substances. Since $\mathrm{X}$-ray fluorescence spectrometry enables the detection of the presence of an element rather than a molecule, it is not possible to distinguish between the prohibited molecules and the others using this analysis technique.

The following hypotheses are used:

- Flame retardants with aluminum and magnesium are identified if the mass percentage of these elements is greater than $60 \%$.

- Talc is identified by the presence of $\mathrm{Mg}$, and kaolin or aluminum silicate by the presence of Al.

Table 2

Tracers of substances associated with the identification of flame retardants and fillers.

\begin{tabular}{|c|c|c|c|}
\hline Tracer & Flame retardants & $\begin{array}{l}\text { Mineral } \\
\text { fillers }\end{array}$ & $\begin{array}{l}\text { Regulated } \\
\text { substances }\end{array}$ \\
\hline $\mathrm{Mg}$ & Magnesium hydroxide & $\begin{array}{l}\text { Magnesium } \\
\text { silicate }\end{array}$ & \\
\hline $\mathrm{Al}$ & $\begin{array}{l}\text { Aluminum trihydroxide } \\
\text { concentration } \approx 60 \%\end{array}$ & $\begin{array}{l}\text { Aluminum } \\
\text { silicate }\end{array}$ & \\
\hline $\mathrm{Si}$ & & Glass fibers & \\
\hline $\mathrm{P}$ & $\begin{array}{l}\text { Phosphonate, phosphinate, } \\
\text { ammonium phosphate, etc. }\end{array}$ & & \\
\hline $\mathrm{Cl}$ & Chlorinated phosphate & & \\
\hline $\mathrm{Ca}$ & & $\begin{array}{l}\text { Calcium } \\
\text { carbonate }\end{array}$ & \\
\hline $\mathrm{Ti}$ & & $\begin{array}{l}\text { Titanium } \\
\text { oxide }\end{array}$ & \\
\hline $\mathrm{Cr}$ & & & $\mathrm{x}$ \\
\hline $\mathrm{Br}$ & PBDE, HBCD, TBBPA & & $\mathrm{x}$ \\
\hline $\mathrm{Cd}$ & & & $\mathrm{x}$ \\
\hline $\mathrm{Sb}$ & Antimony oxide & & \\
\hline $\mathrm{Pb}$ & & & $\mathrm{x}$ \\
\hline
\end{tabular}

\subsection{Density}

The polymer density measurements are important to define the effective density for the wet industrial separation of a mixed fraction of polymers or contaminants.

The sample densities are measured using the immersion method according to the standard (standard ISO 1183-1:2013, 2013). The measurement uncertainty is 0.005 . The measurements are taken on three $200 \mathrm{~g}$ fractions of plastics from the reference sample.

\subsection{Results}

\subsubsection{Contaminant characterization: sorting and shredding defects}

Table 3 presents the mass percentage of polymers and contaminants in the reference sample and the recommendations for separating the contaminants from the plastics. Indeed, paint coating on polymer parts remains a problem for infrared spectrometric detection. Below a certain threshold, paint coating is not a problem for polymer recycling, but it may cause part aspect issues after recycling. As for the remaining links and the other materials, they prevent polymer recycling and remain problematic during recycled plastic transformation. The contaminant rate may be reduced by a finer grinding, which will release the links between polymers. Rubbers and glass can be sorted out by density separation. Foam and cardboard can be extracted by an air classifier due to their very low density. For the remaining metals, magnetic and eddy current sorting are necessary. Finally, green electronic boards can be extracted with color optical sorting. The efficiency of certain sorting technologies may be significantly reduced as particle size decreases.

Photo 2 shows the different links remaining after the shredding step.

\subsubsection{Polymer family characterization}

The results of the Mid-infrared analysis are presented in Fig. 6 .

This characterization makes it possible to define the main plastics to be sorted out for recycling. Given that PC + ABS and PC are chemically compatible, it is possible to group them in a single family (Froelich et al., 2007; Castro et al., 2004/12). The polymers retained for the sorting are HIPS, ABS, PP, PP with fillers and PC (grouped with the $P C+A B S$ ). It should be noted that the ABS family includes SAN and the degraded ABS. Indeed, ABS is constituted of polybutadiene in a SAN matrix. Since polybutadiene deteriorates with age, the infrared spectrum of the ABS is difficult to distinguish from that of SAN because the characteristic bonds of the grouping

Table 3

Mass distribution of plastics and contaminants and recommendations for contaminant extraction $\square$ Contaminants.

\begin{tabular}{lll}
\hline Material & Mass\% & Contaminant extraction solution \\
\hline Plastics & 86.7 & \\
Paint coating & 5.6 & \\
Adhesive labels & 105 & Shredding more finely \\
Plastics / plastics & 2.3 & Shredding more finely \\
Plastics /rubber & 1 & Shredding more finely \\
Plastics/foam & 0.7 & Shredding more finely \\
Plastics/metal & 1.1 & Shredding more finely followed by magnetic \\
& & and eddy current sorting \\
Rubber & 0.9 & Density separation \\
Glass & 0.2 & Density separation and color sorting \\
Foam & $<0.1$ & Air classifier \\
Metal & $<0.1$ & Magnetic and eddy current sorting \\
Cardboard & $<0.1$ & Air classifier \\
Electronic card & $<0.1$ & Color spectrometric sorting \\
Total & 100 & \\
\hline
\end{tabular}




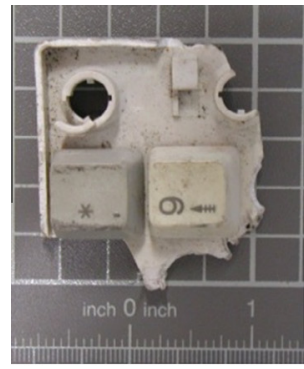

a

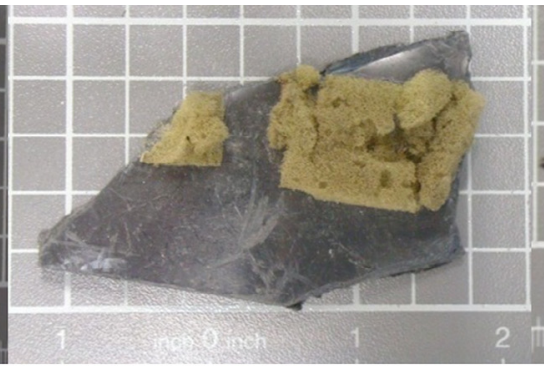

b

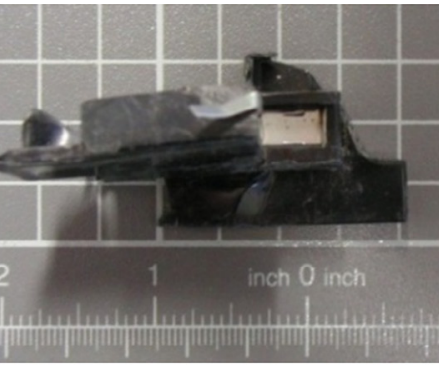

C

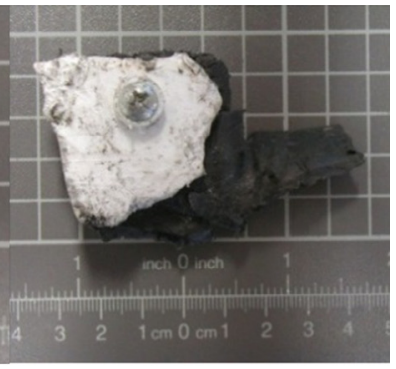

d

Photo 2. (a) Polymer/polymer clipping, (b) polymer/foam sticking, (c) polymer/metal insertion and (d) polymer/polymer/metal screwing.

butadiene do not become any more visible. It is thus very difficult to distinguish between the SAN of the degraded ABS.

\subsubsection{Colors}

The color of the plastics is a determining criterion for plastics sorting with NIR spectrometry. Only light-colored plastics are recognizable with this technology. This technology is suited to plastics that cannot be sorted out by density separation when their densities overlap.

The results of the reference sample color analysis show a distribution of $41 \%$ dark polymers and $59 \%$ light (Fig. 7). The lightcolored polymers can be separated by industrial spectrometric sorting in infrared close relation (NIR). The dark polymers are not discriminated by this technology, because the carbon black used to color the polymers absorbs all of the light. For these categories, other sorting technologies are necessary.

\subsubsection{Fillers and flame retardant: FX identification}

Two samples of each light and dark polymer reduced to powder were analyzed using X-ray fluorescence to determine the concentration of $\mathrm{P}$ (Phosphorus), $\mathrm{Cl}$ (Chlorine), Sb (Antimony), $\mathrm{Br}$ (Bromine), $\mathrm{Cr}$ (Chromium), Cd (Cadmium), $\mathrm{Pb}$ (Lead), MgO (Magnesium oxide), $\mathrm{Al}_{2} \mathrm{O}_{3}$ (Alumina), $\mathrm{SiO}_{2}$ (Silicate), $\mathrm{CaCO}_{3}$ (Calcium carbonate) and $\mathrm{Ti}$ (Titanium).The results show the average percentage of the substances present in the samples.

Fig. 8 shows the results for the elements regulated by the RoHS (Restriction of Hazardous Substances regulation), Fig. 9 those for flame retardants and substances used in synergy $\left(\mathrm{Sb}_{2} \mathrm{O}_{3}\right.$ with $\mathrm{Br}$ compounds) and Fig. 10 those for the mineral fillers.

Fig. 8 shows that cadmium was not detected; its concentration was below 100 ppm (detection threshold). For chromium and lead, the maximum concentration was considerably lower than $0.1 \%$. The bromine concentration exceeded $0.1 \%$ in all of the polymers except the PC and the PP without fillers.

The proportion of both regulated flame retardants, PBB (Polybrominated biphenyls) and PBDE (Polybrominated diphenyl ethers) was not detected by IR or FX spectrometry; only the quantity of bromine $(\mathrm{Br})$ elements was quantifiable. The antimony $(\mathrm{Sb})$ used in synergy with bromine was present in polymers containing bromine, as shown in Fig. 9. Chlorine $(\mathrm{Cl})$ was detected in small quantities (below $0.1 \%$ ) except in the light-colored $A B S(0.25 \%)$, indicating in this case the presence of a chlorinated flame retardant. Phosphorous was detected in considerable amounts in the $\mathrm{PC}+\mathrm{ABS}$. According to the EFRA (European Flame Retardants Association), phosphorous flame retardants are present in $P C+A B S$, in accordance with the results.

The analyzed powder polymers did not contain flame retardant with alumina and magnesium, given the low concentrations (Fig. 10). When polymers contain $\mathrm{MgO}$ and $\mathrm{Al}_{2} \mathrm{O}_{3}$, they also contain $\mathrm{SiO}_{2}$, indicating the presence of magnesium silicate and aluminium silicate. The dark PC + ABS, PC and PP were loaded with calcium carbonate. The HIPS, ABS, SAN, light-colored PC + ABS and lightcolored PC contained almost no mineral fillers.

In conclusion, for the same polymer the substance concentrations were different for the light and dark colors. The signature of titanium oxide used as a white color pigment, is present more strongly in the light-colored polymers. Bromine is more present in the ABS and PC + ABS.

\subsubsection{Density}

The density measurements were performed using three $200 \mathrm{~g}$ samples for each polymer family from the $9 \mathrm{~kg}$ reference sample. The results are presented in Fig. 11. The fraction of floats (density $<1$ ) for the whole $9 \mathrm{~kg}$ batch represents $18 \%$. This fraction containing only polyolefin is significant. The latter can thus be easily separated from the other polymers by density separation.

The PP with fillers, the HIPS, the ABS, the SAN and the PMMA are concentrated in densities that overlap between 1.0 and 1.18. In view of these results, the HIPS, the ABS and the SAN which represent $55 \%$ of the reference sample cannot be sorted out with a density separation process.

$\mathrm{PC}$ and $\mathrm{PC}+\mathrm{ABS}$ are concentrated in densities between 1.19 and 1.22. There are nevertheless some portions of the other polymers in this density range (PA, PP with fillers and ABS).

Above a density of 1.22 , one only finds the PP with fillers and PC, as well as Bakelite and PBT above the density of 1.42 .

\subsection{Characterization methodology discussion}

The characterization of the reference sample according to the criteria seen previously made it possible to identify the main polymers to be recycled in the batch and the quantity of regulated flame retardant. Furthermore, the density measurements provided a first approach to the densimetric distribution of the polymers and made it possible to determine which polymers were potentially separable using density separation. Flame retardants are regulated or prohibited depending on their concentration, their nature and application. Because they are mixed after recycling, the properties of recycled polymers can be deteriorated depending on the flame retardant and its concentration (Riess et al., 2000). The polymers containing flame retardant or fillers can be removed using density separation or X-ray separation (Freegard et al., 2006). The color of polymers is also important, because certain spectrometric sorting technologies do not make it possible to sort out dark-colored polymers.

\section{Conclusion}

One objective of this study was to define a simple characterization methodology to determine the composition of plastics originating from small Waste Electrical and Electronic Equipment (sWEEE), for use routinely by recycling plants. With this aim, a 
batch of 10 tons of sWEEE was sorted, sample items were dismantled and characterized, and the plastics were analyzed. Thanks to this approach, it was possible to estimate the overall polymer composition of the sWEEE. After the 10-ton batch of sWEEE had been ground in an industrial facility, a $9.3 \mathrm{~kg}$ sample of residual plastics was constituted according to the rules and from the collection of ten increments. From this $9.3 \mathrm{~kg}$ sample, only the $+20 \mathrm{~mm}$ fraction was characterized and analyzed; in our case, this fraction represented $5.5 \mathrm{~kg}$.

From the analyzed portion, it was possible to determine the type of polymer, the nature and quantity of flame retardant and fillers (Bromine, Cadmium, Chromium, Lead, Chlorine, Antimony, Silicon, Phosphor, Aluminum, Titanium, and Magnesium), density, color, and the grinding and sorting defects. The polymer types were characterized using Mid-infrared (MIR) spectrometry, whereas the nature and quantity of the flame retardants and fillers were characterized using X-ray fluorescence spectrometry.

The results of the analyses were compared to the estimate of the sWEEE composition and showed that the proposed sampling protocol made it possible to obtain a sample considered representative in terms of its polymer composition. The procedure was validated on a second 10-ton batch of sWEEE collected from another location and treated by a different recycling facility. A compromise between representativeness and analysis time and cost was reached. A quantity of roughly $5.5 \mathrm{~kg}$ of coarse fraction $(+20$ $\mathrm{mm}$ ), corresponding to a $10 \mathrm{~kg}$ primary composite sample and generating about 1500 polymer particles which have to be entirely analyzed one by one, was considered acceptable by all of the project partners.

\section{Acknowledgements}

The authors would like to thank the partners of the TRIPLE (TRI des Polymères) project. Approved by the Axelera cluster, TRIPLE brings together industrial and academic partners: Suez (Environnement) - Sita Group as the leader, EFS technologies, Armines, ARTS (Association de Recherche Technologie et Sciences), Pellenc ST,
MTB Recycling and the BRGM (Bureau de Recherche Géologique et Minière). It was selected by the Unique Inter-ministerial Fund. It is supported by the French State, the Greater Lyon area (Grand-Lyon), the Rhône-Alpes Region and the French ecoorganization "Eco-systèmes".

\section{References}

BIR. Study on the Environmental Benefits of Recycling, 2009.

Castro, M.B.G., Remmerswaal, J.A.M., Reuter, M.A., Boin, U.J.M., 2004. A Thermodynamic Approach to the Compatibility of Materials Combinations for Recycling. Resources, Conservation and Recycling, 2004/12, vol. 43(1), 1-19.

Chancerel, P., Rotter, V.S., 2009. Recycling-oriented characterization of small waste electric and electronic equipment. Waste Manage. 29 (8), 2336-2352.

DIRECTIVE 2002/95/EC of the European Parliament and of the Council of 27 January 2003 on the restriction of the use of certain hazardous substances in electrical and electronic equipment.

DIRECTIVE 2002/96/EC of the European Parliament and of the Council of 27 January 2003 on Waste Electrical And Electronic Equipment (WEEE).

François-Bongarçon, D., 2009. Fishy samples: how big a sample to avoid the infamous 'Poisson effect'?, Fourth World Conference on Sampling \& Blending, The Southern African Institute of Mining and Metallurgy, 43-46.

Freegard, K., Tan, G., Morton, R., 2006. Develop a Process to Separate Brominated Flame Retardants from WEEE Polymers - Final report. Waste \& Resources Action Programme (WRAP), November 2006, 335 p.

Froelich, D., Maris, E., Haoues, N., Chemineau, L., Renard, H., Abraham, F. Lassartesses, R., 2007. State of the Art of Plastic Sorting and Recycling: Feedback to Vehicle Design. Minerals Engineering, 8, 2007, vol. 20(9), pp. 902-912.

Gy, P., 1979. Sampling of Particulate Materials, Theory and Practice. Elsevier, Amsterdam.

Holmes, R.J., 2008. The Importance of Sampling in Grade Control. In: Proceedings of the Sampling 2008 Conference, AusIMM, Perth, Western Australia, Australia, 27-29 May, pp. 77-81.

Huisman, J., Magalini, F., Kuehr, R., Maurer, C., Ogilvie, S., Poll, J., Delgado, C., Artim, E., Szlezak, J., Stevels, A., 2008. 2008 Review of Directive 2002/96 on Waste Electrical and Electronic Equipment (WEEE), Final Report. United Nations University - Study No. 07010401/2006/442493/ETU/G, 347 p.

Riess, M., Ernst, T., Popp, R., Müller, B., Thoma, H., Vierle, O., Wolf, M., van Eldik, R., 2000. Analysis of Flame Retarded Polymers and Recycling Materials. Chemosphere, 6, 2000, vol. 40(9-11), pp. 937-941.

Standard NF 62321 (01/06/2009). Electrotechnical Products - Determination of Levels of Six Regulated Substances (Lead, Mercury, Cadmium, Hexavalent Chromium, Polybrominated Biphenyls, Polybrominated Diphenyl Ethers).

Standard ISO 1183-1:2013. Plastics - Methods for Determining the Density of NonCellular Plastics, 2013 